\title{
Simulation of High Quality Ultrasound Imaging
}

Hemmsen, Martin Christian; Kortbek, Jacob; Nikolov, Svetoslav Ivanov; Jensen, Jørgen Arendt

Published in:

Proceedings of IEEE International Ultrasonics Symposium

Link to article, DOI:

10.1109/ULTSYM.2010.5935765

Publication date:

2010

Link back to DTU Orbit

Citation $(A P A)$ :

Hemmsen, M. C., Kortbek, J., Nikolov, S. I., \& Jensen, J. A. (2010). Simulation of High Quality Ultrasound Imaging. In Proceedings of IEEE International Ultrasonics Symposium (pp. 1739-1742). [5935765] https://doi.org/10.1109/ULTSYM.2010.5935765

\section{General rights}

Copyright and moral rights for the publications made accessible in the public portal are retained by the authors and/or other copyright owners and it is a condition of accessing publications that users recognise and abide by the legal requirements associated with these rights.

- Users may download and print one copy of any publication from the public portal for the purpose of private study or research.

- You may not further distribute the material or use it for any profit-making activity or commercial gain

- You may freely distribute the URL identifying the publication in the public portal

If you believe that this document breaches copyright please contact us providing details, and we will remove access to the work immediately and investigate your claim. 


\title{
Simulation of High Quality Ultrasound Imaging
}

\author{
Martin Christian Hemmsen ${ }^{1,2}$, Jacob Kortbek ${ }^{2}$, Svetoslav Ivanov Nikolov ${ }^{2}$ and Jørgen Arendt Jensen ${ }^{1}$ \\ ${ }^{1}$ Center for Fast Ultrasound Imaging, Department of Electrical Engineering, \\ Technical University of Denmark, DK-2800 Kgs. Lyngby, Denmark \\ ${ }^{2}$ BK Medical, Mileparken 34, DK-2730 Herlev, Denmark
}

\begin{abstract}
This paper investigates if the influence on image quality using physical transducers can be simulated with an sufficient accuracy to reveal system performance. The influence is investigated in a comparative study between Synthetic Aperture Sequential Beamformation (SASB) and Dynamic Receive Focus (DRF). The study is performed as a series of simulations and validated by measurements. The influence from individual element impulse response, phase, and amplitude deviations are quantized by the lateral resolution (LR) at Full Width at Half Maximum (FWHM), Full Width at One-Tenth Maximum (FWOTM), and at Full Width at One-Hundredth Maximum (FWOHM) of 9 points spread functions resulting from evenly distributed point targets at depths ranging from $10 \mathrm{~mm}$ to $90 \mathrm{~mm}$. The results are documented for a 64 channel system, using a 192 element linear array transducer model. A physical BK Medical 8804 transducer is modeled by incorporating measured element pulse echo responses into the simulation software. Validation is performed through measurements on a water phantom with three metal wires, each with a diameter of $0.07 \mathrm{~mm}$. Results show that when comparing measurement and simulation, the lateral beam profile using SASB can be estimated with a correlation coefficient of 0.97 . Further, it is shown that SASB successfully maintains a constant LR though depth at FWHM, and is a factor of 2.3 better than DRF at $80 \mathrm{~mm}$. However, when using SASB the LR at FWOHM is affected by non-ideal element responses. Introducing amplitude and phase compensation, the LR at FWOHM improves from $6.3 \mathrm{~mm}$ to $4.7 \mathrm{~mm}$ and is a factor of 2.2 better than DRF. This study has shown that individual element impulse response, phase, and amplitude deviations are important to include in simulated system performance evaluations. Furthermore, it is shown that SASB provides a constant LR through depth and has improved resolution and contrast compared to DRF.
\end{abstract}

\section{INTRODUCTION}

Ultrasound imaging is an active field of research and each year new imaging schemes are proposed. The performance of these new methods is often documented in an idealized framework, ignoring the complications of real and non-ideal transducers. The consequence of this might lead to a rejection of the new scheme when tested in-vivo.

To gain an insight into how physical transducer characteristics influence the image quality, this paper investigates if they can be simulated to reveal system performance with side-lobe levels down to $-60 \mathrm{~dB}$. The investigation is performed as a comparative study between Synthetic Aperture Sequential Beamforming (SASB) [1] and DRF beamformation.

The study is performed as a series of simulations validated by measurements. The simulations are performed using the Field II [2], [3] simulation software to acquire data and the Beamformation Toolbox III [4] to beamform data.

This paper shows that individual element impulse response, phase, and amplitude deviations are important to include in simulated system performance evaluations. The paper suggests to incorporate measured element pulse echo responses into the simulation sofware as a transducer model that combines excitation waveform and the two-way element impulse response. To emulate a physical 8804 BK Medical 192-element linear array transducer, measured element pulse echo responses are acquired using a BK Medical ProFocus ultrasound scanner.

The rest of this manuscript is organized as follows. Section II presents the measurement system and how the in silico model is created. A brief explanation of the theory behind Synthetic Aperture Sequential Beamforming is also discussed. The results using the in silico model to accurately predict the received echo signal from a multielement focused aperture, is presented and discussed in Section III. This section is followed by conclusions in Section IV.

\section{METHODS}

\section{A. Synthetic Aperture Sequential Beamforming}

The basic idea in multi-element synthetic aperture imaging is to create a pressure wave from multiple elements with a focused transmission. The focal point is introduced as a virtual source, from which a spherical wave emanates within a limited angular region. With SASB a first stage beamformer with a fixed receive time-delay profile and apodization values creates a set of $N$ focused image lines from $M$ emissions. Each point in the focused image line contains information from a set of spatial positions limited by the arc of a circle and the opening angle of the virtual source. A single image point is therefore represented in multiple 1st stage focused image lines obtained from multiple emissions. A second stage beamformer creates a set of high resolution image points by combining information from multiple first stage focused image lines that contain information from the spatial position of the image point.

\section{B. Measurements}

The measurement setup consist of a 2202 ProFocus ultrasound scanner (BK-Medical, Herlev, Denmark) connected to a standard pc through a X64-CL Express camera link (Dalsa, Waterloo, Ontario, Canada) and has previously been described in [5].

The measurement of the individual element pulse echo responses from a BK Medical 8804 linear array transducer is performed as described in [6], where a transducer is mounted in a water tank on a device permitting independent angular adjustment in two orthogonal planes. The target is a stainless steel plate, which dimensions are much larger than the 
transducer beam being measured. This is to avoid multiple reflections from the back surface and sides. The excitation waveform is a bipolar $7.5 \mathrm{MHz} 2$ cycle sinusoid.

The individual element pulse echo time responses are measured by aligning the acoustic surface of the transducer so that the distance from the surface to the steel block is approximately $a^{2} / \lambda, a$ being half the length of the largest dimension of the transducer element and $\lambda$ the wavelength of sound in water at the center frequency.

\section{Simulation}

Simulated data of 9 point targets has been obtained. The points are evenly distributed at depths ranging from $10 \mathrm{~mm}$ to $90 \mathrm{~mm}$. The sampling frequency was set to $120 \mathrm{MHz}$. Specification of simulation parameters can be found in table I. Data was obtained using 3 different simulation setups:

- Idealized: In this setup the transducer element impulse responses are the same across the array. The used element impulse response is aproximated with a measured unit step response from a single element. The excitation waveform is hamming windowed $7.5 \mathrm{MHz} 2$ cycle sinusoid.

- Realistic: In this setup measured element pulse echo responses are used in the simulation software as element excitation that combines excitation waveform and the two-way element impulse response.

- Compensated: This setup is based on the realistic setup, but the measured element pulse echo responses are compensated for amplitude and phase deviations.

\begin{tabular}{ll}
\hline Transducer Parameters (8804) & \\
\hline Pitch & $0.208 \mathrm{~mm}$ \\
Elevation focus & $20 \mathrm{~mm}$ \\
Number of elements & 192 \\
Radius of curvature & linear \\
Element impulse response & delta function \\
\hline Dynamic Receive Focus (SASB) & \\
\hline Focus (Tx / Rx) & $60 \mathrm{~mm} /$ - (10 / 10 mm) \\
F\# (Tx / Rx) & $4.51 / 0.5(2 / 2)$ \\
Apodization (Tx / Rx) & Boxcar / Gauss \\
Apodization 2 nd stage & Hamming \\
\hline Fixed scanner setting & \\
\hline Waveform (idealized) & $7.5 \mathrm{MHz} 2$ cycle sinusoid \\
Waveform (realistic) & element pulse echo \\
Number of emissions / RF lines & $190 / 190$ \\
Maximum no. of active elements & 64 \\
\hline
\end{tabular}

TABLE I

SIMULATION PARAMETERS

\section{Visualization}

The simulated and measured B-mode images were generated by envelope detection using a Hilbert transform, logcompressed to a dynamic range of $60 \mathrm{~dB}$, scan converted and visualized. The images were normalized prior to visualization by calculating the maximum reflection for each of the 9 points targets to determine a normalization curve, so that the maximum reflection for each of the point targets was equal to $0 \mathrm{~dB}$. dB values were mapped linearly to gray scale with 256 shades of gray.

\section{RESULTS}

To address the feasibility of SASB, improved resolution and contrast, the method is first compared in an idealized setup with DRF. Fig. 1 illustrates the simulated B-mode images of 9 point targets using the idealized transducer model (a) DRF and (b) SASB. From Fig. 1(a) it is clearly identified that when using DRF, the lateral resolution and contrast is a function of depth. However, when using SASB the lateral resolution and contrast is much less dependent on depth and a significant improvement compared to DRF is obtained.

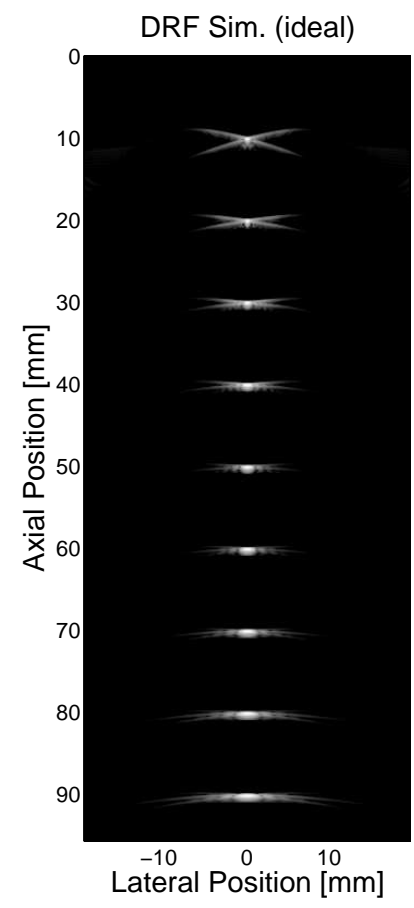

(a) DRF

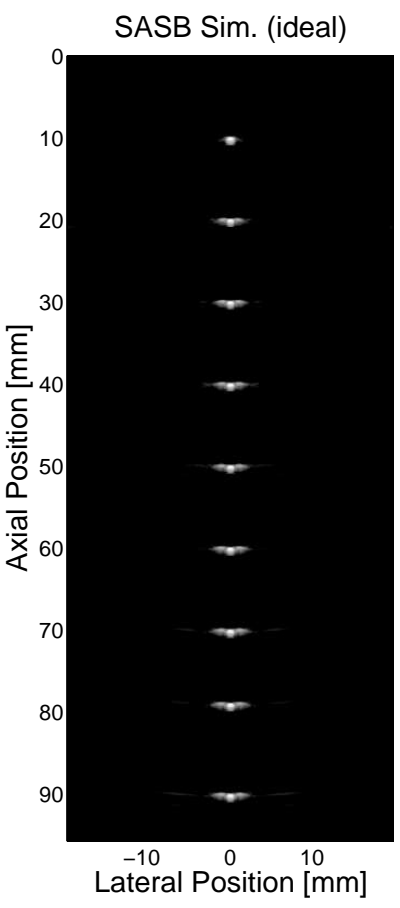

(b) SASB
Fig. 1. Visualization of 9 point spread functions, using (a) DRF beamforming (b) SASB beamforming (idealized transducer model).

System performance can be significantly influenced by the real and non-ideal physical transducer characteristics. To show that simulations can reveal system performance by incorporating element pulse echo responses into the simulation model, the element pulse echo responses are measured and used as a realistic transducer model. The measurement of the array revealed a deviation in the amplitude and a phase error between the elements. Fig. 2 shows the measured amplitude and phase error profile.

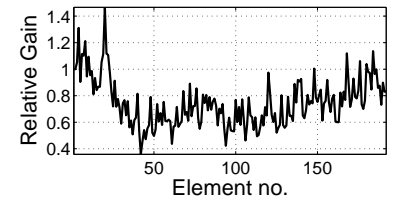

(a)

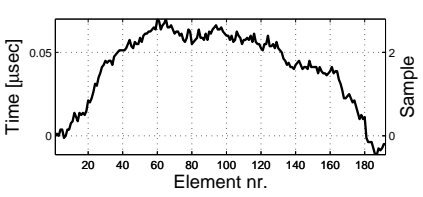

(b)
Fig. 2. (a) Relative amplitude gain and (b) relative phase error

The amplitude has a Coefficient of Variation of 0.24 and the maximum phase error is $0.08 \mu \mathrm{sec}$ or $0.43 \lambda$, determined by finding the largest relative lag though cross-correlation. 
Fig. 3 illustrates the measured element pulse echo responses. In (a) the pulse echo responses for all 192 elements are visualized, black colors indicate negative amplitude and white positive amplitude. Note how the waveform at the edges of the array is offset relative to the center of the array. In (b) the waveform for element no. 10, 96 and 182 are shown. Note how the waveforms at the edges of the array are almost

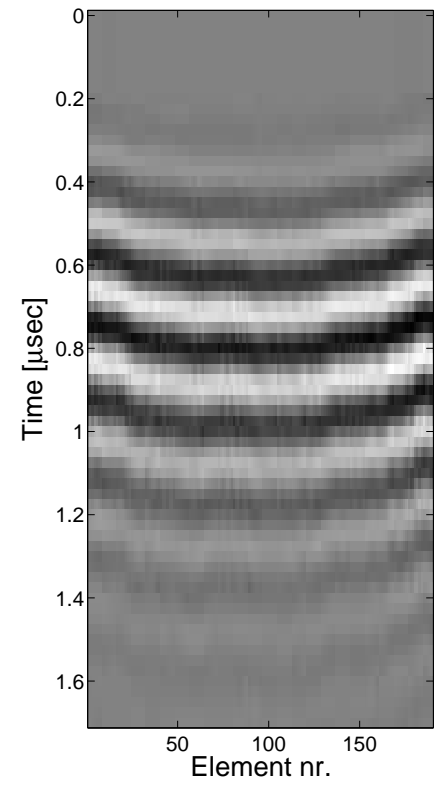

(a)

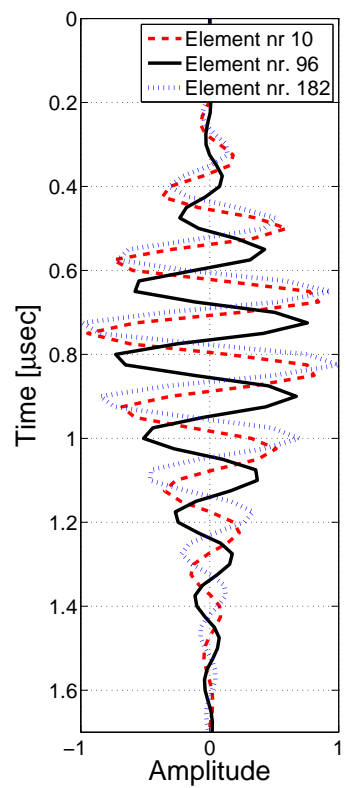

(b)

Fig. 3. Element pulse echo visualized (a) for all 192 elements seen from the top (negative amplitude is black and positive amplitude white) and (b) for element no. 10, 96 and 182 .

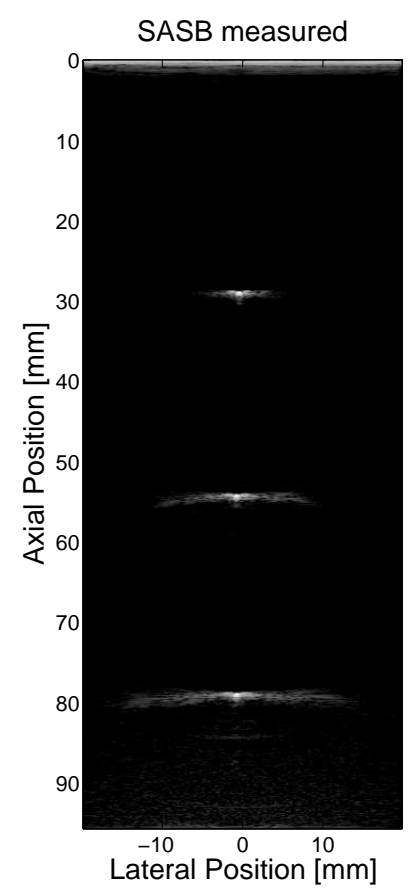

(a)

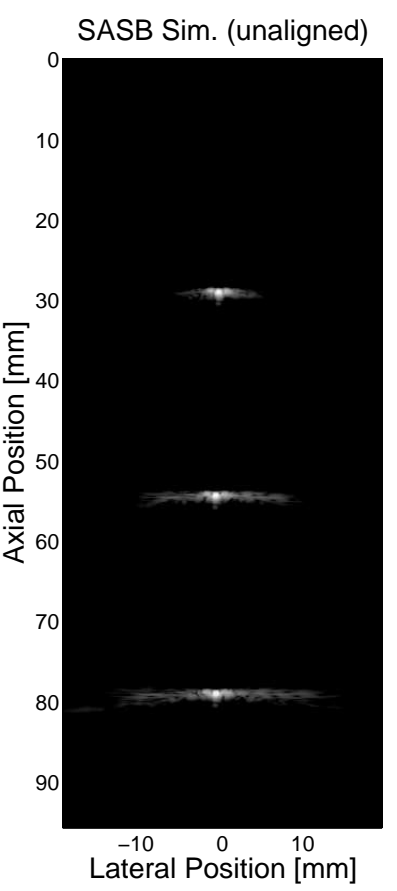

(b)

in phase and have a $154^{\circ}$ phase shift relative to the center element. Fig. 4(a) illustrates the B-mode image generated using measured data beamformed with SASB. Comparing Fig. 4(a) and 1(b) it is clearly seen that using the idealized transducer model the point spread functions does not compare well with measured data. To investigate if the influence on image quality using physical transducers can be simulated with sufficient accuracy, to reveal system performance measured element pulse echo responses are incorporated into the simulation software as a transducer model that combines excitation waveform and the two-way element impulse response. Fig. 4(b) illustrates the simulated B-mode image using SASB for the realistic transducer model. Note how the introduction of inter element deviations in amplitude, phase, and impulse response affect the image quality and how the image compares well with the measured data in Fig. 4(a).

Fig. 4(c) and 4(d) illustrate the simulated B-mode images of the 9 point targets using the realistic transducer model and an amplitude and phase compensated element pulse echo beamformed with (c) SASB and (d) DRF. The amplitude of the waveforms are compensated by normalizing the energy of the $20 \mathrm{~dB}$ most significant part of the waveforms to the energy of element no. 1 , after phase alignment. The phase deviation between the waveforms is compensated by upsampling to 800 $\mathrm{MHz}$ and then using cross-correlation to find the lag with the highest correlation, relative to the waveform of element no. 1 .

Comparing Fig. 1(a) and 4(d), it is clear that use of the compensated transducer model does not noticeably influence the image quality when using DRF. Comparing Fig. 1(b) and $4(c)$ it is observed that the constant resolution as a function of depth is achievable and unaffected by amplitude and phase errors. However, the contrast is seen to be influenced, but much less than when using the uncompensated model.

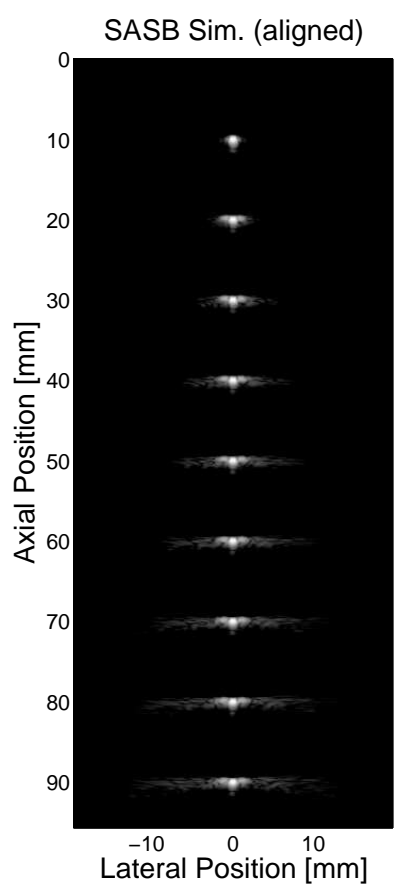

(c)

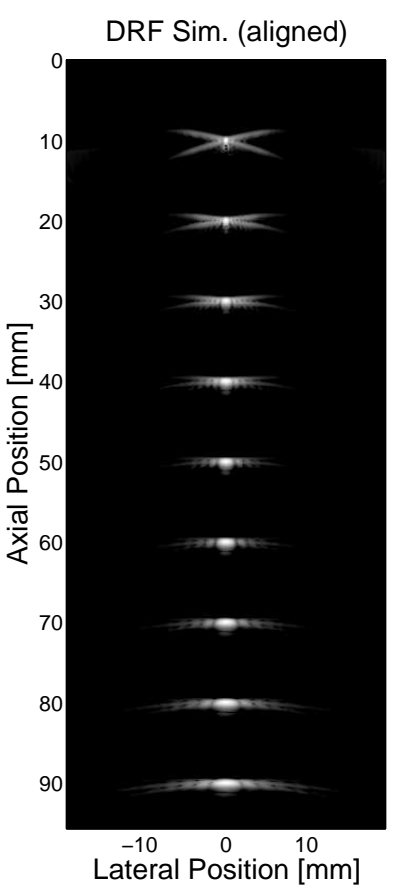

(d)

Fig. 4. Visualization of point spread functions using SASB beamformation (a) measured (b) simulated (realistic transducer model) (c) SASB beamforming (compensated transducer model) and (d) DRF beamforming (compensated transducer model). 
Fig. 5 illustrates the lateral beam profile of the point spread function at $80 \mathrm{~mm}$, beamformed using SASB and measured data, the idealized transducer model, the realistic transducer model, and the compensated transducer model. Note how the 3 models fit with the measured data until $-30 \mathrm{~dB}$. Beyond this level the influence from the transducer model is significant. Clearly having an amplitude and phase aligned transducer is important to achieve good contrast.

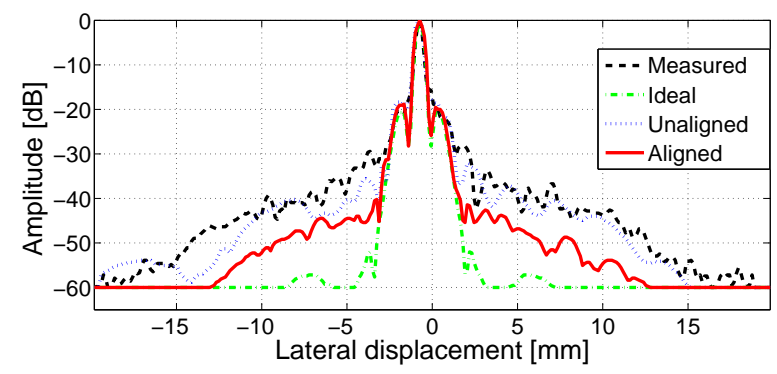

Fig. 5. Lateral cut of the point spread function at $80 \mathrm{~mm}$, using SASB beamforming and measured data, idealized transducer model, realistic transducer model and compensated transducer model

Fig. 6 illustrates the lateral resolution at FWHM, FWOTM and FWOHM using DRF and SASB beamforming using the compensated transducer model. Note how the lateral resolution using DRF expands through depth. Using SASB a constant lateral resolution at FWHM and FWOTM is maintained down to $90 \mathrm{~mm}$. The lateral resolution at FWHM at a depth of 80 mm using SASB is a factor of 2.3 better compared to DRF. At FWOHM the lateral resolution is a factor of 2.2 better then DRF.

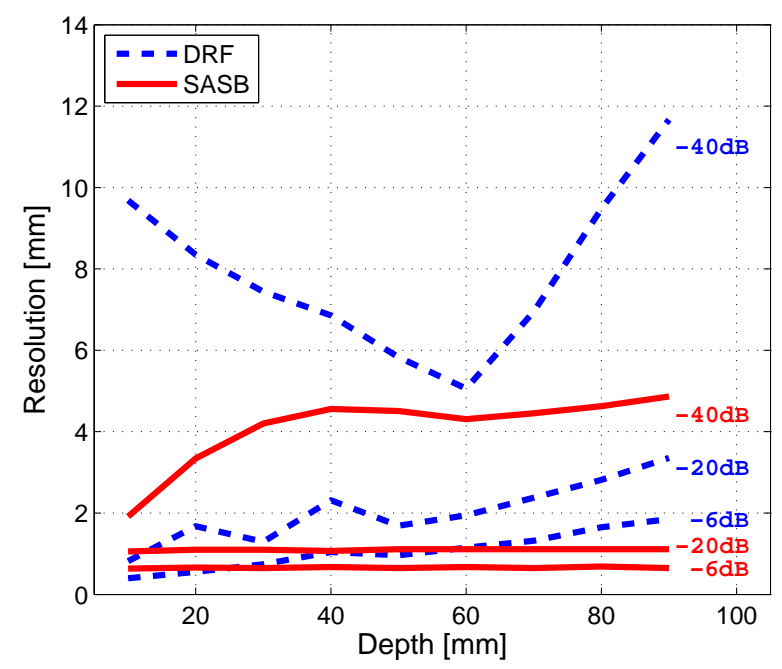

Fig. 6. Lateral resolution at FWHM, FWOTM and FWOHM using DRF (dashed line) and SASB (solid line) beamforming (compensated transducer).

Table II summarizes the lateral resolution using the three different model types and measurements for DRF and SASB beamforming. Values in the table denote the lateral resolution in $\mathrm{mm}$, at FWHM and at FWOHM in parenthesis.

\begin{tabular}{llll}
\hline & \multicolumn{3}{c}{ Depth } \\
\cline { 2 - 4 } Method & $30 \mathrm{~mm}$ & $55 \mathrm{~mm}$ & $80 \mathrm{~mm}$ \\
\hline DRF $_{\text {measured }}$ & $0.6(7.1)$ & $0.9(6.1)$ & $1.5(9.3)$ \\
$\mathrm{DRF}_{\text {unaligned }}$ & $0.7(7.1)$ & $1.0(5.3)$ & $1.6(9.8)$ \\
$\mathrm{DRF}_{\text {ideal }}$ & $0.7(7.2)$ & $1.0(5.4)$ & $1.6(9.2)$ \\
$\mathrm{DRF}_{\text {aligned }}$ & $0.8(7.5)$ & $1.0(4.6)$ & $1.7(9.7)$ \\
$\mathrm{SASB}_{\text {measured }}$ & $0.5(4.4)$ & $0.6(6.8)$ & $0.5(8.8)$ \\
$\mathrm{SASB}_{\text {unaligned }}$ & $0.6(4.7)$ & $0.6(7.3)$ & $0.6(6.3)$ \\
SASB $_{\text {ideal }}$ & $0.6(3.6)$ & $0.6(4.2)$ & $0.6(4.3)$ \\
SASB $_{\text {aligned }}$ & $0.7(3.7)$ & $0.7(4.4)$ & $0.7(4.7)$ \\
\hline
\end{tabular}

TABLE II

SUMMARY OF LATERAL RESOLUTION PERFORMANCE.

\section{CONCLUSIONS}

This paper has shown that the influence on image quality using real and non-ideal transducers can be simulated with an sufficient accuracy, to reveal system performance. Furthermore it is shown that inter element impulse response, phase, and amplitude deviations are important to include in simulated system performance evaluations. The influence on image quality using real and non-ideal transducers characteristics has been investigated in a comparative study between Synthetic Aperture Sequential Beamformation and Dynamic Receive Focus. The study documents the importance of validating high quality imaging systems using realistic simulations, as they reveal the system's performance. Simulations using three different transducer models (idealized, realistic and compensated) show that SASB is more sensitive to element variation than DRF. However, compensating for element phase and amplitude variation, the lateral resolution at FWHM and a depth of 80 mm using SASB is a factor of 2.3 better compared to DRF. At $-40 \mathrm{~dB}$ the lateral resolution is a factor of 2.2 better than DRF. Furthermore when using SASB the lateral resolution can be kept constant though depth.

\section{ACKNOWLEDGMENT}

This work was supported by the Danish Science Foundation and by BK Medical ApS, Denmark.

\section{REFERENCES}

[1] J. Kortbek, J. A. Jensen, and K. L. Gammelmark, "Sequential beamforming - an efficient synthetic aperture technique," IEEE Transactions on Ultrasonics, Ferroelectrics and Frequency Control, Submitted 2010.

[2] J. A. Jensen and N. B. Svendsen, "Calculation of Pressure Fields from Arbitrarily Shaped, Apodized, and Excited Ultrasound Transducers," IEEE Trans. Ultrason., Ferroelec., Freq. Contr., vol. 39, pp. 262-267, 1992.

[3] J. A. Jensen, "Field: A Program for Simulating Ultrasound Systems," Med. Biol. Eng. Comp., vol. 10th Nordic-Baltic Conference on Biomedical Imaging, Vol. 4, Supplement 1, Part 1, pp. 351-353, 1996.

[4] J. M. Hansen and J. A. Jensen, "An object-oriented multi-threaded software beamformation toolbox," Feb. 2011, submitted to SPIE Medical Imaging, 12 -17 February 2011, Lake Buena Vista (Orlando), Florida, USA.

[5] M. C. Hemmsen, M. M. Petersen, S. I. Nikolov, M. B., Nielsen, and J. A Jensen, "Ultrasound image quality assessment: A framework for evaluation of clinical image quality," in Proceedings of SPIE-the international society for optical engineering, vol. 76291. Medical Imaging 2010: Ultrasonic Imaging, Tomography, and Therapy, 2010, pp. 76290C-12.

[6] K. Erikson, "Tone-burst testing of pulse-echo transducers," IEEE Transactions on Sonics and Ultrasonics, vol. 26, no. 1, pp. 7-13, 1979. 